\title{
Editorial from the Editor-in-Chief
}

\author{
Phan Cong Vinh
}

Faculty of Information Technology at Nguyen Tat Thanh University

300A Nguyen Tat Thanh street, Ward 13, District 4, Ho Chi Minh City, Vietnam

\begin{abstract}
Received on 27 November 2019; accepted on 28 November 2019; published on 29 November 2019
Copyright (c) 2019 Phan Cong Vinh, licensed to EAI. This is an open access article distributed under the terms of the Creative Commons Attribution license (http://creativecommons.org/licenses/by/3.0/), which permits unlimited use, distribution and reproduction in any medium so long as the original work is properly cited.
\end{abstract}

doi:10.4108/eai.29-11-2019.163483

On behalf of the Editorial board, we welcome you to the nineteenth issue of the EAI Endorsed Transactions on Context-Aware Systems and Applications. In this issue, we present four selected papers that span various aspects of context-aware systems and applications.

This issue will serve as a reference material for researchers, scientists, professionals and students in computer science and computer engineering as well as developers and practitioners in computing and networking systems design by providing them with state-of-the-art research findings and future opportunities and trends. These papers include some recent advances in context-awareness reflected in this issue. In particular, the issue covers various themes of context-awareness as follows:

Paper 1 by Brita Curum and Kavi Kumar Khedo reports that mobile learning is a new pivotal learning trend nowadays. With the increasing use of sophisticated smartphones equipped with augmented reality supporting tools and sensors, mobile learning platforms are expected to deliver tailor-made and customized learning elements to learners. Contextawareness is regarded as the fundamental approach or workaround to lift this learning style to distribute adaptive and personalized learning elements in mobile devices. The main priority in mobile learning is to make learning elements as flexible as possible using different forms of context data to extend the natural adaptation capabilities in mobile devices in order to engage learners in extremely rich environments. In this paper, a context-aware MoBile LEarning framework is proposed, namely the AMBLE framework. It processes contextual data at four distinct levels namely: Sensing Layer, Adaptation Layer, Context Processing Layer, and Application Layer to perform adaptation of learning contents based on the actual environment and conditions of the learner. Partial implementation of the proposed framework has the potential to capture and represent

${ }^{*}$ Corresponding author. Email: pcvinh@ntt.edu.vn the physical context information that may be used to perform a dynamic adaptation of learning contents and thus significantly improve the mobile learning experiences. Extra work is expected regarding the implementation of the other layers and components of the framework including the user model, context manager, and the adaptation engine. The AMBLE framework proposes some relevant content adaptations with some positive results. As future works, new forms of usercontext adaptation synthesized with other extracted data sets of contextual information will be used to establish and align relevant dynamic adaptation and personalization of learning contents.

Paper 2 by Mien Phuoc Doan, Vu The Tran, Hung Huu Huynh and Hiep Xuan Huynh reports that smart city is a comprehensive application of information resources and a high degree of information technology integration. With the technical support from IoT (Internet of things), smart city needs to have three features of being instrumented, interconnected and intelligent. IoT provides the ability to manage, remotely monitor and control devices from massive streams of real-time data.The model offers a scalable IoT video data analytics applications for smart cities to end users, who can exploit scalability in both data storage and processing power to execute analysis on large or complex datasets. This model provides data analytics programming suites and environments in which developers and researchers can design scalable analytics services and applications. A cloud/edgebased automated video analysis system to process large numbers of video streams, where the underlying infrastructure is able to scale based on the number of camera devices and easy to integrate analytic application. The system automates the video analysis process and reduces manual intervention. Design of the model is developed to be easily extended for new kinds of IoT devices, message routing and queueing, and data analytics, to permit specific application to be programmed via the paradigm to be flexible yet simple. 
Paper 3 by Nguyen Kim Quoc, Vuong Xuan Chi and Nguyen Van Thanh presents that one of the main tasks of controlling communications at the network node is managing the queues, maintaining a reasonable probability of proactive packet removal to minimize congestion, while ensuring the quality of traffic flows and equity in the relationship between traffic flows, when the dynamic state of the network changes. However, current queue management mechanisms still need to be improved to both simplify implementation and improve intelligence in maintaining the average queue length. Therefore, the paper focuses on improving the queue management mechanism at the network node, on the basis of applying the achievements of the soft computing field to supplement the adaptability, the ability to learn, the ability to intelligent decision making for queue management system at the network node.

Paper 4 by Hoang Ngoc Thanh and Tran Van Lang reports that building a good Intrusion Detection System (IDS) model from a certain dataset is one of the main tasks in machine learning. Training multiple classifiers at the same time to solve the same problem and then combining their outputs to improve classification quality is called ensemble method. This paper analyzes and evaluates the performance of using known ensemble techniques such as Bagging, AdaBoost, Stacking, Decorate, Random Forest and Voting to detect DoS (Denial of Service) attacks on UNSW-NB15 dataset, created by the Australian Cyber Security Center 2015. The experimental results show that the Stacking technique with heterogeneous classifiers for the best classification quality with $F$ Measure is $99.28 \%$ compared to $98.61 \%$, which is the best result are obtained by using single classifiers and $99.02 \%$ by using the Random Forest technique.

For the preparation of this nineteenth issue we would like to acknowledge the work of all our Editors, reviewers and authors who have positively supported this publication. We will be happy to receive from our readers any suggestions, including possible proposals for future issues, which may contribute to further maintain the high scientific quality and relevance of this journal.

We hope you will find this nineteenth issue provoking for your research in the field of contextawareness and being useful to your future work.

\section{About the Editor-in-Chief}

Phan Cong Vinh received a $\mathrm{PhD}$ in computer science from London South Bank University (LSBU) in the United Kingdom. He finished his PhD dissertation with the title of "Formal Aspects of Dynamic Reconfigurability in Reconfigurable Computing Systems" at LSBU where he was affiliated with the Center for Applied Formal Methods (CAFM) at the Institute for Computing Research (ICR). At present, he is an Associate Professor of Nguyen Tat Thanh University (NTTU) to take on the responsibility of a senior research scientist. He has been author or co-author of many refereed contributions published in prestigious journals, conference proceedings or edited books. $\mathrm{He}$ is editor of three books titled, "Autonomic Networking-on-Chip: Bio-Inspired Specification, Development and Verification" (CRC Press, 2012), "Formal and Practical Aspects of Autonomic Computing and Networking: Specification, Development and Verification" (IGI Global, 2011) and "Nature-Inspired Networking: Theory and Applications" (CRC Press, 2018). He has served on many conference program committees and has been general or technical (co)chair and (co)organizer of several international conferences such as ICCASA and ICTCC series. His research interests center on all aspects of formal methods in computing, contextawareness, nature of computation and communication, and applied categorical structures in computer science. 